\title{
Refraction-dependent kinematic shift of spectral lines
}

\author{
W.T. Chyla ${ }^{a, b}$ \\ Applied Science Enterprise, P.O. Box 22, 00-975 Warsaw, Poland
}

Received: 1 February 2015 / Revised: 28 February 2015

Published online: 21 April 2015

(C) The Author(s) 2015. This article is published with open access at Springerlink.com

\begin{abstract}
If a luminous source moves with respect to the medium in which it is embedded, the kinematic shift of spectral lines depends both on the relative velocity and on the (relativistic) index of refraction of the medium. This effect is frequency-dependent, which makes it distinguishable from the cosmological redshift, the gravitational redshift and the regular Doppler shift in vacuum, which are all achromatic. The refraction-dependent shift of spectral lines is considered in more detail in the case of Ia supernovae, where the thermally generated electron-positron plasma ball, which expands with relativistic speeds, constitutes the refractive medium; it turns out that the discussed effect is relatively small at the UV and visible frequencies, but it can be significantly larger at longer wavelengths (the IR band). Other examples are given in optics and in other situations, where the refraction-dependent kinematic shift of frequency can be of significance.
\end{abstract}

\section{Introduction}

Spectral shifts of luminous astronomical objects can be affected by a number of phenomena. These include the cosmological redshift, gravity and the Doppler shift due to relative motion of the source of radiation and the observer: orbital motion of the terrestrial observer, peculiar (random) motion of stars, orbital motion of stars in a galaxy, relative motion of galaxies, relativistic motion of luminous baryonic clouds ejected in stellar explosions. Spectral shifts are routinely considered as occurring in vacuum, which is well justified in most, but not in all situations.

In this paper, we consider the kinematic shift of spectral lines due to relative motion of the source of radiation and the refractive medium in which it is embedded. One example of such a situation is the light wave that escapes from the plasma ball spreading with relativistic speeds due to explosion of a supernova. Another example is the light wave that escapes through the oscillating outer plasma layer of a Cepheid. Both types of luminous objects play the role of standard candles.

The shift of frequency due to relative motion of the emitter and the medium is a purely kinematic, specialrelativistic effect and not a dynamic (scattering) phenomenon. Due to dispersion of the refractive index, this type of the kinematic shift of spectral lines is frequency-sensitive, which allows distinguishing this effect from the other, achromatic phenomena that affect frequency of spectral lines (cosmological redshift, gravity, regular Doppler shift in vacuum). Different spectral shifts of different spectral lines have already been observed in spectroscopic binaries and in giant stars [1-3], but the effect considered in this paper is of entirely different nature.

In the next section, we provide the explicit expression for the refraction-dependent kinematic shift of spectral lines in absence of other effects. In the subsequent section, we combine that effect with the cosmological expansion and other effects that contribute to the total redshift of spectral lines. The plasma-enhanced kinematic shift of frequency is exemplified in the special case of the type Ia supernovae (Ia SNs), where extremely high temperatures generate dense electron-positron plasma. A brief summary and a discussion of other situations, where the refraction-dependent kinematic shift of frequency can be of significance, conclude this paper.

\section{Refraction-dependent kinematic shift of frequency}

Let us consider the medium-dependent kinematic shift of frequency in absence of other effects. Figure 1 shows the light wave that is emitted by the source $\mathrm{S}$ and propagates in the expanding cloud of a refractive medium. Next, the

\footnotetext{
a Temporary address: Central Statistical Office, Al. Niepodleglości 208, 00-925 Warsaw, Poland.

b e-mail: chylawt@wp.pl
} 

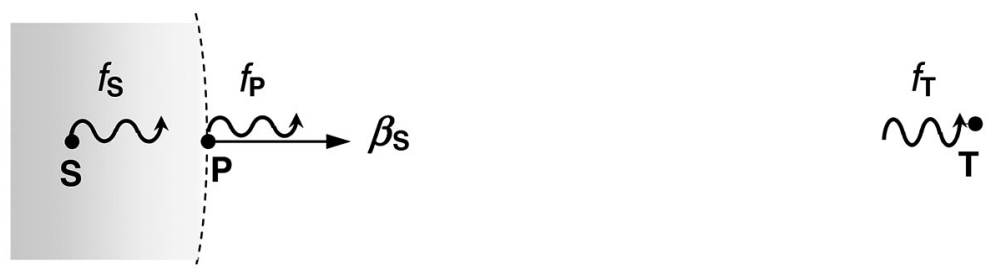

Fig. 1. Frequency of the electromagnetic wave viewed in three reference frames: the proper frame of the source of radiation $\mathrm{S}$, the proper frame of the observer $\mathrm{P}$ on the surface of the medium (gray area), which expands with instantaneous velocity $\beta_{\mathrm{S}}$ (in units of $c$ ) with respect to $\mathrm{S}$, and the proper frame of the observer $\mathrm{T}$. The frequencies are denoted by $f_{\mathrm{S}}, f_{\mathrm{P}}$ and $f_{\mathrm{T}}$, respectively; even though the observers $\mathrm{S}$ and $\mathrm{T}$ are mutually motionless, we have $f_{\mathrm{S}} \neq f_{\mathrm{T}}$.

light wave enters the vacuum (see point $\mathrm{P}$ ) and travels toward the observer $\mathrm{T}$, who is motionless with respect to the source of radiation, $\mathrm{S}$.

We shall make two Lorentz transformations: (a) from the proper frame of the source of radiation, here denoted by $\mathrm{S}$, to the rest frame of the observer $\mathrm{P}$, which sits on the surface of the medium that expands with instantaneous velocity $\beta_{\mathrm{S}}$ (in units of $c$ ) with respect to $\mathrm{S}$ in the line-of-sight towards ${ }^{1} \mathrm{~T}$; and (b) a regular (i.e., in vacuum) Lorentz transformation from the reference frame $\mathrm{P}$ to the rest frame of the observer $\mathrm{T}$. Frequencies of the electromagnetic wave in these three reference frames are denoted by $f_{\mathrm{S}}, f_{\mathrm{P}}$ and $f_{\mathrm{T}}$, respectively.

The Lorentz transformation of frequency from $\mathrm{S}$ to $\mathrm{P}$, which accounts for motion of the medium and refraction, gives [4]

$$
f_{\mathrm{P}}=f_{\mathrm{S}} \frac{1-n_{\mathrm{S}} \beta_{\mathrm{S}}}{\sqrt{1-\beta_{\mathrm{S}}^{2}}}=f_{\mathrm{S}} \frac{\sqrt{1-\beta_{\mathrm{S}}^{2}}}{1+n_{0} \beta_{\mathrm{S}}},
$$

where the exact, special-relativistic expression for the refractive index of the medium in motion is

$$
n_{\mathrm{S}}=\frac{n_{0}+\beta_{\mathrm{S}}}{1+n_{0} \beta_{\mathrm{S}}}
$$

and $n_{0}$ is the refractive index for the light wave in a motionless medium (the tabular value for a given frequency). If $n_{0} \rightarrow 1$ in the emission point, eq. (1) reduces to the regular Doppler shift in vacuum.

The second Lorentz transformation from $\mathrm{P}$ to $\mathrm{T}$ (in vacuum) is straightforward and gives

$$
f_{\mathrm{T}}=f_{\mathrm{P}} \sqrt{\frac{1+\beta_{\mathrm{S}}}{1-\beta_{\mathrm{S}}}}=f_{\mathrm{S}} \frac{1+\beta_{\mathrm{S}}}{1+n_{0} \beta_{\mathrm{S}}} .
$$

Equations (1) and (3) show that

a) the refraction-sensitive kinematic shift of frequency depends on the refractive index $n_{0}$ at the point of emission $\mathrm{S}$, and does not depend neither on the length $L$ of the path ${ }^{2}$ nor on the refractive index $n(\boldsymbol{r})$ further along the path of the light wave in the (transparent) medium: once the frequency of the emitted light wave is transformed to the rest frame of the medium, its frequency stays the same irrespective of $L$ and possible rarefaction of the medium $\left(n_{0}(\boldsymbol{r}) \rightarrow 1\right)$, which can change the wavelength $\lambda$, but not the frequency $f$ of the wave;

b) if the medium at the emission point is rarefied $\left(n_{0} \rightarrow 1, n_{\mathrm{S}} \rightarrow 1\right)$, the medium-dependent shift of frequency reduces to the regular Doppler effect in vacuum;

c) in the case of a non-ionized medium $\left(n_{0}>1\right)$ the observer $\mathrm{T}$ registers lower frequency than the emission frequency, i.e., $f_{\mathrm{T}}<f_{\mathrm{S}}$ (redshift), even though $\mathrm{S}$ and $\mathrm{T}$ are mutually motionless;

d) in the case of an ionized medium $\left(0<n_{0}<1\right)$ the observer T registers higher frequency, i.e., $f_{\mathrm{T}}>f_{\mathrm{S}}(\mathrm{blueshift})$;

e) the occurrence of redshift or blueshift depends also on the direction of flow of the medium relative to the wave vector of the light wave (the sign of $\beta_{\mathrm{S}}$ );

f) since $n_{0}$ is dispersive, the refraction-dependent shift of spectral lines is implicitly frequency-dependent;

g) the shift is independent of the distance from the observer $\mathrm{T}$.

Due to a) and g), the refraction-dependent kinematic shift of frequency is a local effect.

\footnotetext{
${ }^{1}$ Most of radiation reaches the observer $\mathrm{T}$ from the central area of the luminous body; radiation from the visible edge is less intense and its shift of frequency is less than the one computed for the line-of-sight direction, which contributes to the asymmetric broadening of the spectral line.

${ }^{2}$ Provided that $L \gg \lambda$, so that the medium can be treated as bulk matter.
} 
The corresponding parameter $z_{\mathrm{m}}$ is

$$
z_{\mathrm{m}}=\frac{\lambda_{\mathrm{T}}-\lambda_{\mathrm{S}}}{\lambda_{\mathrm{S}}}=\frac{f_{\mathrm{S}}-f_{\mathrm{T}}}{f_{\mathrm{T}}}=\frac{\beta_{\mathrm{S}}\left(n_{0}-1\right)}{1+\beta_{\mathrm{S}}},
$$

where $\lambda$ denotes the wavelength measured in reference frames indicated by subscripts S or T, respectively. The subscript "m" indicates that the "medium" plays a key role in this kinematic effect.

The refraction-dependent kinematic shift of spectral lines of astronomical objects has not been considered earlier. At high velocities of the refractive medium relative to the source of radiation $\mathrm{S}$, calculations require using the exact, relativistic index of refraction, which has been derived only recently [4].

\section{Merging the refraction-dependent kinematic shift of frequency with other phenomena that affect frequency of spectral lines}

The medium-dependent kinematic shift of spectral lines is a local phenomenon, which is entirely independent of the cosmological expansion that goes on while light is travelling in vacuum toward the terrestrial observer. Therefore, the observed frequency $f_{\text {obs }}$ is

$$
f_{\mathrm{obs}}=f_{\mathrm{S}} \frac{1+\beta_{\mathrm{S}}}{1+n_{0} \beta_{\mathrm{S}}} \cdot \frac{a_{\mathrm{S}}}{a_{\mathrm{obs}}},
$$

where $a_{\mathrm{S}}$ and $a_{\mathrm{obs}}$ are cosmological scale factors at the time of emission and observation of the light wave, respectively. Having denoted the cosmological redshift parameter as

$$
z_{\mathrm{c}}=\frac{a_{\mathrm{obs}}}{a_{\mathrm{s}}}-1
$$

and the observed value of the redshift parameter as

$$
z_{\mathrm{obs}}=\frac{\lambda_{\mathrm{obs}}-\lambda_{\mathrm{S}}}{\lambda_{\mathrm{S}}}=\frac{f_{\mathrm{S}}}{f_{\mathrm{obs}}}-1
$$

eq. (5) can be expressed in the equivalent form

$$
\left(z_{\mathrm{obs}}+1\right)=\left(z_{\mathrm{c}}+1\right)\left(z_{\mathrm{m}}+1\right)
$$

or

$$
z_{\mathrm{obs}}=z_{\mathrm{c}}\left(z_{\mathrm{m}}+1\right)+z_{\mathrm{m}}
$$

If $z_{\mathrm{m}}<0$ (blueshift), the observed value of $z_{\mathrm{obs}}$ is less than the purely cosmological redshift $\left(z_{\mathrm{obs}}<z_{\mathrm{c}}\right)$; if $z_{\mathrm{m}}>0$ (redshift), the refraction-dependent effect increases the total redshift $z_{\mathrm{obs}}$.

Other independent phenomena, which affect frequency $\left(e . g .\right.$, gravity, macroscopic ${ }^{3}$ motion of the source of radiation with respect to the terrestrial observer T) enter the expression for the observed frequency $f_{\text {obs }}$ as factors, so that the total observed redshift $z_{\mathrm{obs}}$ can be factorized

$$
\left(z_{\mathrm{obs}}+1\right)=\left(z_{\mathrm{c}}+1\right)\left(z_{\mathrm{m}}+1\right)\left(z_{\mathrm{D}}+1\right)\left(z_{\mathrm{g}}+1\right)(\ldots),
$$

where $z_{\mathrm{D}}$ accounts for the regular Doppler shift (in vacuum) due to relative motion of the terrestrial observer with respect to the source of radiation, $z_{\mathrm{g}}$ denotes the gravitational redshift ${ }^{4}$ and $(\ldots)$ stands for possible other effects.

\section{Magnitude of the refraction-dependent shift of frequency in the case of type la SNs}

According to eq. (4), the theoretically allowed range of blueshifts $z_{\mathrm{m}}$ is rather wide

$$
-0.5<z_{\mathrm{m}} \leq 0
$$

\footnotetext{
${ }^{3}$ The microscopic (thermal) motion of a large number of sources $\mathrm{S}$ contributes to the symmetric broadening of the spectral line.

${ }^{4}$ Where extreme gravity enters the picture, the kind of clocks must be explicitly specified. In the case of extreme gravity combined with refraction, the set of mutually motionless standard clocks (atomic clocks) does the job [5,6]. In this paper, we do not consider radiation emitted by so extremely dense objects.
} 
The lower bound on $z_{\mathrm{m}}$ corresponds to $n_{0} \rightarrow 0$ and $\beta_{\mathrm{S}} \rightarrow 1$; the upper bound corresponds to $n_{0} \rightarrow 1$ (vacuum). The physically realistic magnitudes of $z_{\mathrm{m}}$ can be narrowed to

$$
-0.09<z_{\mathrm{m}} \leq 0
$$

because $\beta_{\mathrm{S}} \approx 0.1$ in Ia SN explosions. Moreover, plasma of very high density $\left(n_{0} \rightarrow 0\right)$ is opaque, so that the refractive index of the medium must be non-zero, and actually quite close to 1 to ensure that the medium is transparent; this further narrows the range of possible values of $z_{\mathrm{m}}$.

To estimate the value of $z_{\mathrm{m}}$ in the case of type Ia SNs, we need to determine the magnitude of the refractive index $n_{0}$ of such a system. Let us use a simple model [7] for $n_{0}$ of the electron plasma ${ }^{5}$ at a given frequency $f$

$$
n_{0}=\sqrt{1-N e_{0}^{2} /\left(4 \pi^{2} \varepsilon_{0} m f^{2}\right)}=\sqrt{1-f_{\text {plasma }}^{2} / f^{2}}
$$

where $e_{0}$ is the elementary charge, $\varepsilon_{0}$ is permittivity of vacuum, $m$ is the electron mass, $N$ is the electron density and $f_{\text {plasma }}$ is the plasma frequency.

The maximum temperature $T$ of the $\mathrm{SN}$ explosion approaches $10^{10} \mathrm{~K}$, i.e., thermal energy $k_{\mathrm{B}} T$ is on the order of $1 \mathrm{MeV}$; at so high temperatures the electron-positron plasma is generated. The characteristic time of creationannihilation processes is $10^{-10} \mathrm{~s}$, which is very short compared with the rate of change of global physical conditions during the SN explosion. Thus, the $\mathrm{e}^{-} \mathrm{e}^{+}$plasma density is determined by local temperature at a given instant. The equilibrium density of $\mathrm{e}^{-} \mathrm{e}^{+}$plasma at temperatures $\sim 10^{10} \mathrm{~K}$ is $10^{36}-10^{37}$ particles per $\mathrm{m}^{3}$. So extremely dense plasma is opaque and its spectrum is continuous, with no discernible contribution from the characteristic atomic spectra. Atomic spectral lines become visible only when temperature gradually drops and density of thermally generated plasma is gradually reduced due to recombination, so that it becomes transparent. The electron plasma is transparent for light of visible and near-IR frequencies at densities $N=10^{26} \mathrm{~m}^{-3}$; at this point the refraction-dependent shift of spectral lines enters the picture. Further decrease in temperature and density of plasma (down to the levels determined by ionization of atoms) results in $n_{0} \rightarrow 1$ and gradual vanishing of the refraction-dependent shift of frequency. In other words, the refraction-dependent shift of spectral lines is a temporary phenomenon, which can be observed near the maximum of brightness, in the presence of dense, thermally generated plasma.

Existence of thermally generated electron-positron plasma in SN explosions can be observationally confirmed by detecting a strong $1 \mathrm{MeV}$ pulse that corresponds to the annihilation process. At lower temperatures and lower densities of the electron-positron plasma, bound states of these particles (positronium) can be investigated by spectroscopic means, because the positronium spectrum is well known [8]. The visible-range spectral lines (e.g., the 5.1 eV transition $1^{1} \mathrm{~S}_{0} \rightarrow 2^{1} \mathrm{~S}_{0}$ at rest) may be difficult to detect due to the high intensity of the continuous background radiation; the hyperfine spectral line corresponding to the transition $1{ }^{1} \mathrm{~S}_{0} \rightarrow 1^{3} \mathrm{~S}_{0}$ in the microwave range $\left(2.0338 \times 10^{11} \mathrm{~Hz}[9]\right.$ at rest $)$ is the preferred choice for detecting the thermally generated electron-positron plasma, due to a higher signal-to-noise ratio in this range of frequencies.

As density of the electron-positron plasma gradually decreases, it becomes transparent to UV, visible and IR frequencies, so that atomic spectral lines can get through the plasma ball toward the terrestrial observer. Therefore, let us assume that the density of particles is $N=10^{26} \mathrm{~m}^{-3}$ to ensure transparency of plasma in the whole range of the considered spectrum, $1 \times 10^{14} \mathrm{~Hz}-1 \times 10^{15} \mathrm{~Hz}$, which covers visible frequencies $\left(4 \times 10^{14} \mathrm{~Hz}-8 \times 10^{14} \mathrm{~Hz}\right)$, as well as near-UV and near-IR.

The computed values of $z_{\mathrm{m}}$ are given in fig. 2 for $\beta_{\mathrm{S}}$ equal to $0.10,0.03$ and 0.01 . It shows that the refractiondependent blueshift is

$$
-0.05 \lesssim z_{\mathrm{m}} \leq 0
$$

at near-IR. The blueshift is very small in the UV range, but it increases significantly at lower frequencies $\left(z_{\mathrm{m}}=\right.$ -0.00037 at the near-UV frequency $f=1 \times 10^{15} \mathrm{~Hz} ; z_{\mathrm{m}}=-0.0015$ at the visible frequency $f=5 \times 10^{14} \mathrm{~Hz}$; $z_{\mathrm{m}}=-0.051$ at the near-IR frequency $f=1 \times 10^{14} \mathrm{~Hz}$, at $\beta_{\mathrm{S}}=0.1$ ) and the shift can acquire even higher magnitudes at lower IR frequencies ${ }^{6}$.

Let us compare the computed values of the blueshift $z_{\mathrm{m}}$ with the observed values of the redshift $z_{\mathrm{obs}}$ for Ia SNs and the uncertainty of $z_{\mathrm{obs}}$. The values of $z_{\mathrm{obs}}$ for "nearby" Ia SNs, which were used as standard candles for calibration purposes, are in the range of 0.01-0.1. The acceleration of the cosmological expansion and existence of dark energy that fuels this process were inferred from observations in the $z$-range of $0.1-1[10,11]$, and the highest values of $z_{\text {obs }}$ for Ia SNs exceed $z_{\mathrm{obs}}=1.7[12]$.

\footnotetext{
${ }^{5}$ Contribution of the heavy component of plasma to the refractive index is much less due to much larger mass of such particles. The collision correction is also negligible in the first order approximation, at densities that ensure transparency of plasma at visible, near-IR and near-UV frequencies.

${ }^{6}$ On the other hand, transparency of plasma decreases at longer wavelengths, which means that such spectral lines are more difficult to observe.
} 


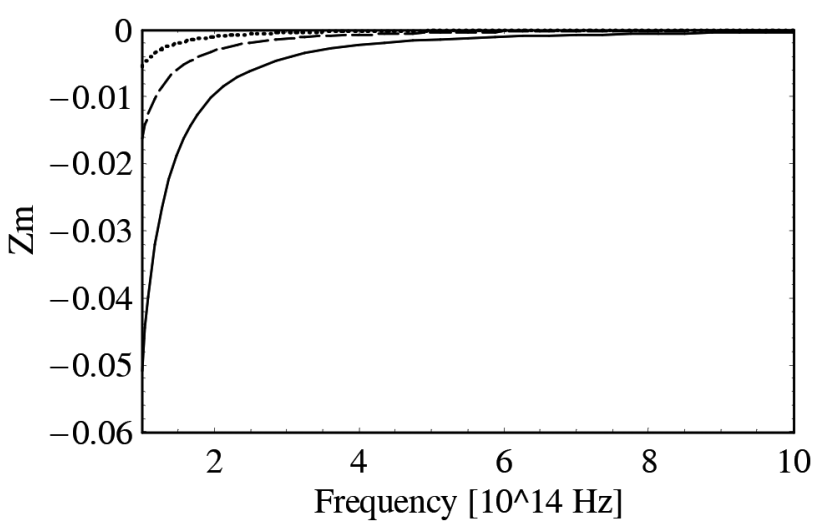

Fig. 2. The refraction-dependent shift of spectral lines, $z_{\mathrm{m}}$, in the frequency range $1 \times 10^{14} \mathrm{~Hz}-1 \times 10^{15} \mathrm{~Hz}$, shown at three different speeds of the plasma ball: $\beta_{\mathrm{S}}=0.10$ (continuous line), $\beta_{\mathrm{S}}=0.03$ (broken line), and $\beta_{\mathrm{S}}=0.01$ (dotted line). The assumed plasma density is $N=10^{26} \mathrm{~m}^{-3}$.

The uncertainty of observed redshifts is usually in the range of $\Delta z_{\text {obs }}=0.001-0.01[11,12]$; the lower limit corresponds to the estimates of the peculiar velocity of $\sim 300 \mathrm{~km} / \mathrm{s}$, which is actually unknown, and the upper limit is similar to $\Delta z_{\mathrm{obs}}$ of host galaxies. The uncertainties $\Delta z_{\mathrm{obs}}$ are relatively small and do not cause much concern. The principal source of uncertainty in the Hubble diagram for Ia SNs is the uncertainty associated with brightness of high- $z$ supernovae, which is determined from observational data with the use of complex analytical and statistical tools. Therefore, some Hubble diagrams for Ia SNs show the uncertainty bars associated with brightness, but not the uncertainty bars for redshifts, see, e.g., fig. 1 on p. 11 in [10].

The magnitude of the refraction-dependent contribution $z_{\mathrm{m}}$ to the value of $z_{\mathrm{obs}}$ in the crucial region of $z_{\mathrm{obs}}>0.1$ in the visible band is relatively small, similar to the uncertainty associated with the (unknown) peculiar motion of the luminous source; however, the refraction-dependent correction can be significant for spectral lines in the IR band. The Hubble diagram for Ia SNs shows some astray Ia SNs [10,11], which are blueshifted by as much as -(0.1-0.2), relative to what is expected based on the redshift $v s$. brightness relationship in various cosmological models ${ }^{7}$. Some of these outliers were included into the population of the analysed Ia SNs, and some were screened out, based on the probable role of the galactic dust that dims the luminous source.

Since the contribution of $z_{\mathrm{m}}$ to the total value of $z_{\mathrm{obs}}$ hinges on a significant density of thermally generated $\mathrm{e}^{-} \mathrm{e}^{+}$ plasma, existence of such plasma in Ia SNs should be confirmed observationally before $z_{\mathrm{m}}$ is included into the analysis. Simultaneous observation of the $1 \mathrm{MeV}$ peak and the microwave signal, as well as temporal variation of intensity and broadening of these signals can provide direct insight into physical properties of the plasma ball generated by the SN explosion. Such observations are difficult, because they should be performed above the atmosphere and because supernova explosions are one-time events that are not predictable. On the positive side (1) the temporal evolution of thermally generated electron-positron plasma should be similar for all Ia SNs; and (2) the subject of correlation between $\gamma$ and microwave signals is interesting by itself.

\section{Summary and discussion}

Let us briefly summarize a few distinct features of the refraction-dependent kinematic shift of spectral lines: a) it is frequency-sensitive due to dispersion of the refractive index (i.e., frequency shifts of different spectral lines of the same luminous source can be different), which makes it qualitatively distinguishable from the other effects that are achromatic; b) since $z_{\mathrm{m}} \sim f^{-2}$, in the first-order approximation ${ }^{8}$, the refraction-dependent shift of spectral lines is larger and easier to observe at lower frequencies, i.e., in the IR band rather than in the visible or UV range; c) in rapidly changing objects, such as novae, supernovae and Cepheids, density and other properties of the refractive medium do change with time, which implies temporal variability of $z_{\mathrm{m}}$ and consequently variability of $z_{\mathrm{obs}}$; d) the sign of $z_{\mathrm{m}}$ changes with the sign of $\beta_{\mathrm{S}}$ (the direction of relative motion), just as in the regular Doppler effect in vacuum; e) for a given sign of $\beta_{\mathrm{S}}$, the sign of $z_{\mathrm{m}}=\beta_{\mathrm{S}}\left(n_{0}-1\right) /\left(1+\beta_{\mathrm{S}}\right)$ in plasma $\left(n_{0}<1\right)$ is different from the sign of $z_{\mathrm{m}}$ in the non-ionized refractive medium $\left(n_{0}>1\right)$, which is quite different from the regular Doppler effect in vacuum, where the relative velocity is the only important parameter.

\footnotetext{
7 Even if the brightness of those astray Ia SNs were "corrected up" by the maximum amount of its uncertainty, their redshift would still be off by about -0.1 (blueshift) from what is expected.

8 This relation can be perturbed by the varying optical depth of the medium, which involves local density of particles and frequency of the light wave.
} 
The IR spectra of "nearby" objects are preferred for testing the contribution of $z_{\mathrm{m}}$ to the total redshift $z_{\mathrm{obs}}$, because a) the observed line shifts of such objects are less dominated by the cosmological redshift; b) spectra of "nearby" objects are of much better quality; and c) the effect is much stronger at longer wavelengths.

The refraction-dependent kinematic shift of frequency can occur wherever a luminous object and its refractive envelope are in relative motion. The natural candidate systems for such tests are novae and supernovae in our Galaxy or in the Local Group, especially the type Ia SNs. This is because $z_{\mathrm{m}}$ as a function of frequency and time is expected to be similar for all Ia SNs: due to the same intrinsic luminosity of Ia SNs, homogeneity of their spectra that imply the same chemical composition, and the same mechanism of explosion (upon reaching the threshold mass due to accretion), one can expect that properties of the plasma ball and its temporal evolution is analogous for all ("nearby" and distant) type Ia SNs.

Another type of standard candles, Cepheids, are also preferred systems for detection and investigation of the refractive effect in line-shifts. This is due to the known mechanism of pulsation, periodicity that allows to eliminate non-systematic occurrences (random perturbations) and the high helium content of the ionized outer layer, which reminds chemical composition of Ia SNs.

The key signature of the refraction-dependent kinematic shift of spectral lines is detection of multiple frequency shifts in the spectrum of a single luminous source. However, one should take into account that: a) multiple shifts of spectral lines can also occur in spectra of giant stars due to different optical depths [1]; b) there are numerous double redshift objects, $z_{1} \neq z_{2}$, which are interpreted as a merger of two objects (stars, quasars or galaxies) that cannot be otherwise resolved $[2,3]$; c) three different sets of frequency shifts $z_{1} \neq z_{2} \neq z_{3}$ in a single luminous source can be viewed as a triple merger [13]. It would not be reasonable, however, to explain many different frequency shifts of spectral lines of a single luminous source with a multiple merger model (provided that spectral lines are correctly identified); in that case, dependence of $z_{\text {obs }}$ on frequency can be interpreted as a non-negligible contribution of the refraction-dependent kinematic shift of frequency $z_{\mathrm{m}}$ to the observed value of $z_{\mathrm{obs}}$.

The medium-enhanced kinematic shift of frequency is relevant in all situations where the source, the medium and the observer are in mutual motion. The key quantity that determines magnitude of this effect is the product $n \beta$, which should be compared with $\beta$ in vacuum. Therefore, the refraction-dependent kinematic effect is significant: 1) at highly relativistic speeds, which is illustrated by the example considered in this paper; 2) in situations where the system is highly frequency-sensitive, e.g., the slow light phenomenon in a vibrating, electromagnetically induced transparent medium (the group velocity of light in the medium in motion can be derived directly from the relativistic index of refraction $n$ [14]; 3) in situations that require utmost accuracy, e.g., in defense applications of the GPS and in radar systems, where the time of propagation and the frequency shift of the emitted and reflected wave depend explicitly on $n[15]$.

Open Access This is an open access article distributed under the terms of the Creative Commons Attribution License (http://creativecommons.org/licenses/by/4.0), which permits unrestricted use, distribution, and reproduction in any medium, provided the original work is properly cited.

\section{References}

1. S.A. Alexeeva, A.M. Sobolev, S.Yu. Gorda, M.V. Yushkin, V. McSwain, Astrophys. Bull. 68, 169 (2013) DOI: 10.1134/S1990341313020041.

2. P. Tsalmantza, D.W. Hogg, Astrophys. J. 753, 122 (2012) DOI: 10.1088/0004-637X/753/2/122.

3. T.A. Boroson, T.R. Lauer, Nature 458, 53 (2009) DOI: 10.1038/nature07779.

4. W.T. Chyla, Optik 124, 1477 (2013) doi.org/10.1016/j.ijleo.2012.04.012.

5. W.T. Chyla, Can. J. Phys. 78, 721 (2000) DOI: 10.1139/p00-023.

6. W.T. Chyla, Can. J. Phys. 78, 755 (2000) DOI: 10.1139/p00-021.

7. V.L. Ginzburg, Propagation of Electromagnetic Waves in Plasma (Gordon and Breach Science Publishers, New York, 1962) chapt. 2, ISBN-10: 0677200803, ISBN-13: 978-0677200804.

8. D.H. Perkins, Introduction to high energy physics, 3rd edition (Addison-Wesley, Menlo Park, CA, 1987) sect. 5.14, ISBN 0-201-12105-0.

9. C. Itzykson, J.B. Zuber, Quantum field theory (McGraw-Hill, New York, 1980) sect. 10.3, ISBN 0-07-032071-3.

10. Scientific background on the Nobel Prize in physics 2011. The accelerating universe, compiled by the Class for Physics of the Royal Swedish Academy of Sciences, 4 October 2011,

http://www.nobelprize.org/nobel_prizes/physics/laureates/2011/popular-physicsprize2011.pdf.

11. S. Perlmutter et al., Astrophys. J. 517, 565 (1999) DOI: 10.1086/307221.

12. D. Rubin et al., Astrophys. J. 763, 35 (2013) DOI: 10.1088/0004-637X/763/1/35.

13. X. Liu, Y. Shen, M.A. Strauss, Astrophys. J. Lett. 736, L7 (2011) DOI: 10.1088/2041-8205/736/1/L7.

14. W.T. Chyla, J. Electromagn. Waves Appl. 27, 938 (2013) DOI: 10.1080/09205071.2013.792752.

15. W.T. Chyla, Can. J. Phys. 78, 747 (2000) DOI: 10.1139/p00-022. 